\title{
Narcissisme et processus de civilisation
}

\section{Pierre-Henri Castel}

Quand un historien et un philosophe de la psychiatrie et de la psychanalyse rencontre le problème de la diffusion d'une catégorie «psy », le narcissisme, dans des jugements de personnalité réputés significatifs, voire exemplaires dans une configuration sociale donnée, il se pose plusieurs questions. La plus spontanée relève l'écart de qualité conceptuelle entre le narcissisme comme classification ordinaire et le narcissisme des psychanalystes et des psychologues - ce qui implique une critique non moins sévère des usages semi-savants et semi-vulgarisés du narcissisme par les médiateurs culturels, autrement dit celui des moralistes, dans la veine de la lecture de Lasch par Richard Kilminster qui ouvre ce numéro. De ce point de vue, je vais le montrer, le scepticisme à l'égard de la notion de «culture du narcissisme » peut encore augmenter sans passer par le constat de sa fragilité en termes sociologiques, en mettant simplement en valeur la quasi-vacuité de sa prétention à expliquer psychologiquement quoi que ce soit. Mais faut-il en rester à la vision intellectualiste d'une déperdition du contenu scientifique de l'idée de narcissisme, au fur et à mesure qu'elle se popularise comme catégorie évaluative du discours commun ? Car, cette déperdition, qui en doute ? L'enjeu, c'est plutôt d'examiner comment son appropriation comme «idée sociale» (ce que je distingue d'une idée sociologique) suit des étapes spécifiques, et développe des potentialités de sens et d'usage qui étaient pour une part implicites dans le concept savant, en y sélectionnant certains traits au détriment d'autres, mais pas au hasard. Plus difficile encore, c'est de découvrir comment cette appropriation soutient et parfois même précède son usage savant, en dévoilant plus ou moins un certain besoin social qui se dégage dans le fait d'y recourir dans les jugements interpersonnels. En somme il faudrait fournir, idéalement, ce qu'une approche strictement intellectualiste du concept de narcissisme serait bien en peine de fournir dans le lexique usuel de la critique épistémologique : des circonstances d'usage et des conséquences d'application, en sorte que les coordonnées sociales et historiques contribuent substantiellement au sens, y compris savant, de la catégorie de narcissisme.

Insister sur ce dernier point, c'est d'ailleurs assumer une certaine distance à l'égard des facilités qui invoquent le fait, dans la modernité récente, d'une "culture psychologique de masse », pour reprendre l'expression de Robert Castel. Sauf à supposer les masses hypnotisées par de grands mots, il faudrait en effet inclure dans le tableau de cette culture ses relais pratiques concrets. Je pense notamment à l'expérience (de plus en plus fréquente statistiquement) de la consultation psychothérapeutique dans nos sociétés. Car cette expérience peut être vue comme un lieu où les gens rencontrent le bien-fondé (éventuel) des catégories «psy», ou encore les reçoivent de la bouche de ceux qui en ont le maniement autorisé, et ils le réfléchissent ensuite dans le système de leurs prénotions non sans en évaluer certaines raisons d'être, produisant alors la catégorie " psy » dite populaire. En tout cela, les individus se montrent actifs, voire réflexifs. Ceux qui ont eu, si peu que ce soit, personnellement ou par procuration (amis, famille), l'occasion d'en tester la signification en psychothérapie, manipulent, certes, encore une prénotion ; mais on ne peut plus ramener à une pure illusion ce qu'ils disent, grâce à elle, de l'esprit de leur société.

Hélas, il n'existe aujourd'hui pas grand-chose en matière de sociologie de la psychothérapie, et plus généralement d'études empiriques du malaise personnel et du «travail sur soi » dans les sociétés actuelles, ainsi que de leurs répercussions sur les institutions, qui ait les lettres de créance de la sociologie de la religion après Weber, quand elle s'est intéressée à l'articulation soigneuse du contexte socio-historique, des dogmes, des pratiques collectives et intimes, ainsi que des mutations concomitantes des structures sociales et des structures psychiques. Or c'est quelque chose de ce genre ce qu'il nous faudrait pour dépasser le simple constat de la psychologisation envahissante des rapports sociaux. (De fait, la psychothérapie a repris nombre de fonctions assumées autrefois par les expériences spirituelles, même si elle en a médicalisé et donc naturalisé une bonne partie.) 
Il y aurait encore bien d'autres questions préalables à soulever, j'en reste à ces deux premières, ainsi qu'à ma remarque sur l'impasse où nous sommes, faute d'avoir approché la sociologie de la psychothérapie avec les mêmes moyens et surtout les mêmes ambitions que la sociologie de la religion. Trois chantiers au moins, en tout cas, paraissent dignes d'intérêt, pour qui ambitionnerait une lecture vraiment sociologique de la catégorie de narcissisme.

En premier lieu, extraire de Freud ce qui, dans sa conception inaugurale de la notion, offre certaines potentialités à la critique morale de l'individualisme - mais en entrant dans la texture fine de son concept, pour effectivement créditer ceux qui s'en emparent à cette fin d'authentiques raisons de se l'approprier, et en tâchant de garder le contact avec les pratiques psychothérapeutiques concrètes qui le mobilisent, dans leurs apories et leurs paradoxes les plus importants.

En second lieu, interroger l'écart criant qui se manifeste entre la conception freudienne du narcissisme et toute une série de reprises ultérieures de la notion. Car une partie de ces reprises revendiquent de le réélaborer psychanalytiquement, ce qui serait motivé par diverses exigences de la clinique, mais aussi par l'évolution des mœurs, tandis qu'une autre abandonne en fait toute référence explicative à un cadre freudien ou post-freudien, et fait du narcissisme un ensemble comme un autre de traits de personnalité, support éventuel d'un «trouble de la personnalité narcissique ». Que signifie cette divergence ? Et que veut dire la tentative de la résorber en invoquant néanmoins Freud à tous les tournants, alors que le narcissisme de la «culture du narcissisme » entretient un rapport en réalité ténu avec le concept original. J'essaierai en tout cas de montrer que c'est cette seconde version du narcissisme, post- comme non-freudienne, qui a inspiré et qui inspire toujours la critique morale de l'individualisme (celle de Lasch, ou celle d'autres essayistes plus récents ${ }^{1}$ ).

En troisième lieu, spéculer sur ce que la sociologie d'Elias, et tout particulièrement la façon dont elle articule autocontrainte et surmoi freudien, pourrait apporter à une compréhension authentiquement sociologique du narcissisme (plus précisément, d'une dimension narcissique dans la «société des individus », qui fasse droit aussi bien à certaines caractéristiques savantes de la notion, héritées de Freud qu'au besoin social qui se manifeste dans nos évaluations interpersonnelles). Car il y a bien chez Freud une relation étroite entre narcissisme et surmoi (médiée par la notion d'idéal du moi). Mais cette relation intrapsychanalytique est-elle transposable dans la reconstruction sociohistorique du surmoi par Elias ? En un mot, peut-on faire du narcissisme un concept éliasien par analogie avec la façon dont il a relu le surmoi freudien au prisme de l'autocontrainte ? Finalement, toutes ces considérations permettent-elles de compléter la suggestion de Kilminster que, sous couvert de «culture du narcissisme », on a psychologisé (et déformé) un ensemble de phénomènes qui serait bien mieux défini sociologiquement par référence à l'informalisation théorisée par le sociologue hollandais Cas Wouters?

\section{Le narcissisme de Freud est-il vraiment celui des individus «narcissiques »?}

Face à une pensée aussi évolutive que celle de Freud, il est plus utile de se concentrer sur les fonctions que jouent dans ses arguments les concepts-clés, comme celui de narcissisme, que sur des définitions dogmatiques - qui, en plus, font perdre de vue les circonstances pratiques de leurs redéfinitions successives à mesure que l'œuvre se déploie ${ }^{2}$. De ce point de vue, le narcissisme de Freud n'est jamais un observable clinique, mais un méta-concept qui revêt deux rôles théoriques, dont l'articulation est tout sauf évidente.

Le premier, c'est d'être un opérateur d'unification des auto-érotismes, autrement dit, des multiples

\footnotetext{
${ }^{1}$ On peut penser, en France, à Jean-Claude Michéa, dans sa préface à la seconde traduction française de La Culture du narcissisme (Paris, Flammarion, 2006), mais aussi à Philippe Muray. Lasch a été pris au sérieux par des auteurs aussi variés que Marcel Gauchet, Gilles Lipovetsky ou Anthony Giddens, comme si s'y référer était incontournable dans toutes les critiques morales contemporaines de l'individualisme.

${ }^{2}$ Renvoyer à Bourdin...
} 
modalités pulsionnelles du rapport au corps propre instauré dès l'enfance. À cet égard, le narcissisme résulte du passage au statut de substantif des divers «auto- » des auto-érotismes. De tous ces «auto-», on tire un moi-plaisir, dit Freud. C'est ainsi que le narcissisme exprime la sexualisation de l'autoconservation de l'organisme, alors que Freud, longtemps, distinguait les pulsions sexuelles des pulsions d'autoconservation. Mais le narcissisme devient ainsi un opérateur de totalisation psychologique en général. Par exemple, la différence entre identification narcissique et identification hystérique, c'est que la première est intégrale (je deviens « tout comme » l'objet), tandis que la seconde ne porte que sur des traits spécifiques de l'objet auquel on s'identifie (par exemple sa manière de sourire).

Le second rôle théorique du narcissisme, une fois consommé le passage de l' « auto- » au substantif, c'est-à-dire la libidinalisation du moi, c'est de désigner un stade du développement psychosexuel, voué au dépassement, où l'on passerait de l'amour de soi à l'amour d'objet. Il importe énormément à Freud que ce passage soit réversible, et qu'on puisse donc régresser au narcissisme, par exemple dans le deuil consécutif à la perte de l'objet d'amour. Car c'est uniquement dans l'examen des phénomènes de régression qu'il peut y avoir une clinique du narcissisme. En effet, ce qui est pleinement et radicalement auto-érotique (narcissique) reste refoulé, étant donné qu'il y a toujours à un degré ou à un autre de l'extériorité et de l'objet : à proprement parler, il ne peut donc pas y avoir d'expérience consciente du narcissisme. C'est la raison pour laquelle, au grand scandale des tenants des théories de la séduction «réelle » des enfants par leurs parents ${ }^{3}$, Freud a soutenu que la plupart des attentats sexuels dans l'enfance dont se rappellent les gens sont en réalité la retraduction d'expériences auto-érotiques dans le lexique ultérieur, plus développé, des rapports aux objets. Et donc le séducteur adulte du souvenir est comme surajouté à une expérience masturbatoire infantile devenue tout à fait impensable. Simplement, ces fantasmes de séduction ont le même indice de réalité psychique que les expériences objectivement vécues.

Cette référence à l'infantile est décisive pour comprendre l'exploitation psychosociologique du narcissisme chez Freud. Car, dans le schéma évolutionniste dominant à l'époque, il tient bien sûr aussi que les primitifs en sont encore au narcissisme. D'où l'importance, chez eux, de la «pensée magique », c'est-à-dire de l'action directe du psychique sur le monde extérieur. Par contraste, le processus de civilisation implique toujours une renonciation à l'auto-érotisme : c'est la condition sine qua non pour que la pulsion sexuelle devienne exploitable socialement.

Dans la clinique et la thérapeutique, qu'est-ce que cela signifie ? On ne s'en rend compte, je viens de le dire, qu'en déclinant les différentes figures de la régression narcissique. Relèvent ainsi de ladite régression l'enfoncement dans le sommeil, qui sert de fondement à l'«égoïsme » des représentations oniriques (le rêve, selon Freud, n'a qu'un seul personnage, le rêveur lui-même) ; mais aussi l'effondrement mélancolique, comme perte pure, jusqu'au suicide, de l'amour que le moi se porte à soi-même (comme s'il était alors l'objet de son deuil). L'homosexualité masculine est plus qu'exemplaire de l'analyse psychologique en termes de narcissisme. Sans entrer dans les détails, il y a de bonnes raisons de soupçonner qu'elle y a joué un rôle moteur. Freud veut éviter, à la différence des sexologues de son époque, qu'on postule une pulsion homosexuelle spécifique. La sexualité homosexuelle masculine n'est absolument pas une sexualité « autre ». C'est une sexualité où un trait narcissique du moi masculin, la présence auto-érotique du pénis, continue d'être projeté sur l'objet et devient la condition sine qua non de l'amour qu'on lui porte. Sans ce moi-pénis en miroir, pas d'amour. Mais dans tous les cas de figure de cette régression narcissique, et quelles qu'en soient les modalités pathologiques, la notion de narcissisme a en fait une portée relationnelle. Le narcissisme sert à indexer les bases inconscientes de la difficulté, voire de l'incapacité au transfert : car dans l'amour de transfert, opérateur décisif de la cure, il faut bien que l'autre puisse exister comme objet. En sorte que les névroses «narcissiques », de façon paradigmatique la mélancolie, ou encore l'auto-érotisme radical de la paranoïa (où tout tourne autour du malade et de

\footnotetext{
${ }^{3}$ C'est un lieu commun des Freud Wars, depuis Jeffrey Moussaieff Masson, The Assault on Truth : Freud's Suppression of the Seduction Theory, Farrar, Straus \& Giroux, 1984.
} 
ses ressentis subjectifs), constituent des obstacles insurmontables à une thérapie par la psychanalyse. Soit le patient est indifférent, enfermé en soi; soit, au contraire, il projette massivement sur le psychanalyste, ce qui peut aller jusqu'à un sentiment de persécution devant ses interprétations, ou à un attachement érotomaniaque, c'est-à-dire à une certitude délirante d'être aimé de lui. (Je conjecture que ces accidents, nullement rares, ont solidifié la conviction pratique de la pertinence de la notion de narcissisme, à mesure que les cures se multipliaient.)

Mais outre ces significations cliniques, le narcissisme a aussi une valeur plus caractérologique, et un sens moral nettement plus intuitif et surtout détachable du système complexe des hypothèses de la psychanalyse, et tout à fait indépendamment de la thérapie. Ce sont les divers passages où Freud a une vision si j'ose dire «baudelairienne » de l'autosuffisance psychologico-morale de certains individus : ainsi de la femme qui se sait belle, laquelle évoque les chats et les grands fauves libres et inéducables, mais aussi les gens pleins d'humour que «rien n'atteint» des malheurs objectifs qui les frappent, voire certains grands criminels. Insistons bien: le narcissique peut être, parfois, conscient d'être narcissique, mais sa conscience de soi n'est pour rien dans la force de l'amour qu'il se porte. Ce sont ces tableaux qui ont, semble-t-il, connu une vraie postérité, en renouvelant les clichés des moralistes : la dénonciation de l'égoïsme des individus, ou celui de l'amour-propre caché dans les conduites altruistes. Voyez notamment son portrait du «type narcissique »: un leader novateur, se tenant lui-même en haute estime, peu intimidable mais qui, surtout, préfère aimer qu'être aimé. J'y reviendrai.

La transition avec la psychologie sociale est alors naturelle. C'est là où convergent les aspects unitifs-totalisants du narcissisme par rapport à la vie pulsionnelle (à la multitude des autoérotismes) et ses aspects relationnels, autrement dit ses potentialités régressives sur le chemin qui conduit à l'objet, c'est-à-dire à autrui. La notion charnière est celle d'idéal du moi. Car c'est toujours sur une totalité extérieure que se projette en effet la complétude illusoire (car perdue) d'un organisme radicalement déchiré par le jeu conflictuel de ses pulsions. Cette totalité est notamment la figure idéale d'un père. La seule consistance que le moi individuel puisse avoir, c'est celle d'un moi idéal qui est comme le «reflet» de cet idéal du moi externe, sinon transcendant. Mais il se produit alors un renversement capital. Une fois cette instance de l'idéal du moi effectivement incarnée, par exemple dans la figure d'un individu du «type narcissique » décrit plus haut, diverses propriétés de la psychologie des foules s'éclairent. En particulier, surgit la possibilité que l'amour narcissique de chaque individu qui la compose «vide» leur moi au profit de cet idéal qui les hypnotise, jusqu'au sacrifice de leur vie pulsionnelle, et finalement de leur vie tout court, en faveur de l'être aimé. L'objet se met alors à la place de l'idéal du moi, suggère Freud, comme si chacun pouvait de cette façon s'aimer à la façon dont le leader de la foule s'aime lui-même.

Il est habituel, enfin, de repérer dans cette constellation d'idées un certain nombre de tensions, voire de contradictions. Je n'en signale qu'une seule, car c'est celle qui a eu le plus d'importance pour les controverses entre post-freudiens. Elle tient à ceci que, dès l'introduction du concept de narcissisme, on l'a vu, le moi est le réservoir de la libido (au principe de l'auto-érotisme). Freud, en 1914, est sans équivoque sur ce point. À peine dix ans plus tard, cependant, en 1923, ce n'est plus le moi, mais le ça. Au contraire, le moi désexualise la libido d'objet, celle qui jaillit originairement de l'inconscient pulsionnel, la transformant en libido narcissique. D'une certaine manière, ce renversement était prévisible. Car, si l'on y réfléchit, il est implicite dans l'idée d'un rapport à soi inconsciemment sexualisé. En 1923, c'est cet implicite qui tient désormais le premier rôle. Mais ce renversement ouvre les deux avenues dans lesquelles s'engouffreront les successeurs de Freud. Soit le «soi » (héritier du moi de l'auto-érotisme) prime sur la vie pulsionnelle, et ce qui compte alors dans le narcissisme est la relation idéalisante à autrui, lequel est mis en fonction comme un objetmiroir - moyennant quoi ce soi est susceptible d'intégrer la vie pulsionnelle. Soit, au contraire, le moi reste à jamais marqué par l'illusion de la complétude — auquel cas la vie pulsionnelle empêche toute réconciliation avec l'objet, quel qu'il soit. Tout dépend, en somme, des deux accentuations possibles d' « auto- » et d' « érotismes ». 
En tout état de cause, même une présentation à très gros traits de la problématique freudienne du narcissisme signale plusieurs éléments directement antagoniques à l'idée qu'on s'en fait d'ordinaire ${ }^{4}$. Chez Freud, il n'est jamais accessible réflexivement. Même la projection narcissique sur l'idéal du moi échappe radicalement au moi, ce qui en fait, si «lucide » se croit-il, le jouet de n'importe quelle foule aimantée par un leader. Le narcissisme ne saurait non plus être une modalité d' «expression» de soi (car il est sans dehors); et il ne procure aucune volupté coupable quand l'égoïsme pulsionnel s'y déchaîne pourtant au détriment objectif d'autrui (car, justement, on n'atteint pas le stade où autrui serait constitué comme tiers à léser). Et il n'est de toute façon pas directement observable, puisqu'il est inféré d'une clinique de la régression narcissique, hors de laquelle il n'a aucun sens.

\section{La «personnalité narcissique » et son « trouble » une controverse américaine}

Comment donc le narcissisme freudien a-t-il pu devenir une ressource critique pour les moralistes consternés par l'impact psychologique de l'individualisme des sociétés modernes ? L'impression générale est en effet que le narcissisme freudien stricto sensu renvoie à une sorte d'asocialité innocente, à un «hors-jeu » qui ne peut justement pas avoir de signification relationnelle. Si l'on ne se contente pas de répondre qu'une idée fausse peut bien avoir n'importe quel antécédent logique, il faut alors se risquer à quelques hypothèses sur les principes de sélection qui ont privilégié tel ou tel trait du concept original, pour aboutir au nouveau narcissisme de la «culture du narcissisme ». Encore une fois, une approche purement épistémologique ne suffit pas. Tout ce qu'elle peut faire, c'est indiquer des pistes pour une future étude de sociologie de la connaissance. Car ce qu'on sait depuis les travaux d'Alain Ehrenberg, c'est que les représentations de la personne dans leur contexte politique et historique conditionnent les idées psychologiques du moi et du soi, ce qui apparaît de façon éclatante quand on compare les destins intellectuels de la psychanalyse en France et aux États-Unis 5 .

Par exemple, la détestation lacanienne pour la «psychologie du moi » annafreudienne longtemps dominante aux États-Unis peut bien se présenter comme le résultat d'une exégèse plus rigoureuse de Freud; elle atteste aussi, et peut-être surtout, une incompréhension profonde devant une vie sociale où le self, loin d'être le résultat atomisé d'un collectif malade de l'individualisme libéral, est au contraire une institution au sens fort, un principe de solidarité. À cet égard, il y a une certaine ironie à voir les essayistes qui se plaignent de l'atomisation individualiste des sociétés néolibérales face à la multiplication des comportements "narcissiques», se saisir de Lasch, un penseur explicitement conservateur pour qui le narcissisme est une maladie de l'individualisme américain traditionnel, celui des personnalités «fortes »! $!^{6}$ En tout état de cause, ces comparaisons France/États-Unis envoient un signal d'alerte : s'il y a bien des raisons épistémologiques internes au développement et à la «dégénérescence » du narcissisme freudien, il est futile de croire qu'elles sont le fin mot de l'histoire, et qu'on peut faire l'impasse sur les normes de la construction sociale de la personne dans des contextes historiques précis. C'est ce que je suggérais en parlant des attentes sociales touchant certaines catégories «psy ». Ces attentes ne se situent pas simplement en

\footnotetext{
${ }^{4}$ J'ai considérablement simplifié. Pour plus de détails, le lecteur pourra par exemple se reporter à la notice très fouillée de Dominique Bourdin dans le Dictionnaire freudien dirigé par Claude Le Guen, Paris, Presses universitaires de France, 2008, p. 931-962.

${ }^{5}$ Alain Ehrenberg, La Société du malaise. Le mental et le social, Paris, Odile Jacob, 2010.

${ }^{6}$ Chez Lasch, la nostalgie pour le «rugged individualism » à la Hoover, cet esprit de l'homme libre qui ne compte que sur lui-même, est évidente. Pourtant, il existe en effet toute une «critique sociale » d'inspiration psychanalytique qui a recyclé Lasch en l'utilisant plutôt comme un témoin, significatif pour la clinique, de la déchéance de la subjectivité néolibérale. Ce courant de pensée, en outre, ne tient pas compte de ceci que Lasch se présente plutôt comme le passeur de la clinique psychanalytique du narcissisme auprès des sociologues ou des politistes, nullement comme un critique de la société qui puisse inspirer la psychanalyse ! Ces croisements et ces malentendus sont frappants chez des essayistes comme Jean-Pierre Lebrun, Charles Melman ou Dany-Robert Dufour, qui se réclament tous de Lacan.
} 
aval, au niveau de la récupération populaire des discours savants, mais aussi en amont dans leur genèse, et même dans la formalisation de leur pertinence scientifique.

Ceci posé, il ne fait aucun doute que le narcissisme mobilisé dans la polémique morale à partir des années 1980, chez Lasch, lui est parvenu filtré par un certain nombre de controverses savantes internes à la psychanalyse, mais explicitement post-freudiennes, et dont, pour autant que je puisse voir, ceux qui s'en sont plus tard inspiré n'ont aucune idée.

Revenons en effet sur les deux manières d'accentuer l'auto-érotisme qui sont à la racine du concept freudien de narcissisme. Deux écoles vont s'opposer à partir des années 1960 aux États-Unis. Pour la première, emmenée par Heinz Kohut, l'accent doit porter prioritairement sur le soi, au sens où la cure doit avoir pour objectif pratique de réparer les sentiments d'identité vacillants. La psychanalyse freudienne n'avait échoué à traiter les patients narcissiques que parce qu'elle niait ou dévalorisait certaines formes d'empathie réglée qui constituent le thérapeute comme un objet réparateur des perturbations du moi du patient. Jusqu'à un certain point, les idées de Kohut étaient compatibles avec l'orthodoxie psychanalytique annafreudienne, dont une lecture rustique mais répandue faisait du moi « fort »du psychanalyste un appui et un modèle pour celui du patient. Mais le recours à l'empathie, et l'idée que l'objectif de la psychothérapie est de développer un «sens du soi » à partir d'objets internalisés qui s'appuient en miroir dans le transfert sur le self du thérapeute, aboutissent plus ou moins à évacuer les problèmes de la vie pulsionnelle.

Confrontée aux mêmes patients au «moi fragile », prompts à passer à l'acte, sans estime d'euxmêmes, ou surcompensant par des attitudes grandioses suivis d'effondrements dépressifs, une école concurrente se développe sous la houlette d'Otto Kernberg. Elle prend les choses de manière diamétralement opposée. Elle conteste la désexualisation rampante du narcissisme à la Kohut, et y voit un déni de l'agressivité pulsionnelle. Elle considère les choix d'objet narcissique non pas comme un appui salutaire, mais comme une potentialité pathologique dans laquelle c'est un soi infantile qui est projeté sur l'objet.

La controverse a fait rage pendant plusieurs années, chaque école considérant l'autre comme un danger public ${ }^{7}$. Il y a donc une certaine ironie, à nouveau, à voir Lasch, qui puise l'essentiel de sa documentation chez Kernberg, invoquer ponctuellement Kohut comme si l'accord sur l'existence de «troubles narcissiques » dispensait de l'examen des divergences sur leur interprétation et leur traitement. Kernberg, qui a popularisé les notions de troubles narcissiques et de troubles borderline (états-limites), a finalement emporté la lutte, ce qui a conduit à l'expulsion des partisans de la selfpsychology des instances internationales de la psychanalyse. Les tenants et aboutissants de cette querelle, du moins aux États-Unis, disent quelque chose de l'esprit de l'individualisme de l'époque. D'une façon tout à fait non freudienne, Kohut introduit en effet une dimension d'expressivité positive dans le self, faisant peu ou prou du narcissisme, s'il est tempéré et ajusté à autrui, un trait de normalité épanouie. Or il s'ensuit une conséquence remarquable, dès lors que le narcissisme n'est plus ce qui apparaît dans une régression toujours pathologique en puissance : c'est une nouvelle tolérance à l'homosexualité - qui était le cas-type de la fixation à un moi-à-pénis narcissiquement projeté sur le partenaire désirable. Peu importe, en fait, que Kohut ait été lui-même homosexuel (dans une autobiographie à peine déguisée, il évoque sa séduction, adolescent, par son précepteur). Le déplacement de l'intérêt de la thérapie vers la meilleure « intégration » du self, quelles que soient les vicissitudes sous-jacentes de l'auto-érotisme, n'est pas passé inaperçu dans le paysage des psychothérapies de l'époque. On pouvait réparer le moi tout en se passant enfin de la théorie des pulsions, laquelle avait toujours paru bizarre.

Mais il s'agit là d'une première divergence touchant le narcissisme. Une seconde se fait jour à peu près au même moment. La psychologie de la personnalité, alors très vivante aux États-Unis, a tenté de mettre tout le monde d'accord sur la base d'inventaire de traits de personnalité obtenue par des questionnaires standardisés. Existe-t-il, ainsi, une personnalité narcissique ? Peut-on, sur cette base

\footnotetext{
${ }^{7}$ On trouvera des renseignements beaucoup plus étoffés dans André Renaud, «A propos du narcissisme », Filigrane, vol. $20, \mathrm{n}^{\circ} 1,2011$, p. 57-74, et vol. $20, \mathrm{n}^{\circ} 2,2011$, p.55-85.
} 
à la fois tempéramentale, caractérologique et relationnelle, spécifier de façon objective en quoi consiste un «trouble de la personnalité narcissique »?

Je me contenterai d'indications tout aussi cursives ${ }^{8}$. Mais le débat dure depuis plus de trente ans. Plusieurs éléments s'en dégagent. Le premier, c'est que, s'il existe une telle personnalité, elle a moins de crédibilité psychométrique que les autres (par exemple les personnalités schizoïdes ou obsessionnelles). Elle est traversée par une tension qui la subdivise en général en au moins deux sous-types : un type «grandiose» et un type «vulnérable» (je privilégie ces descriptions pour qu'elle fasse écho à la polémique entre Kohut et Kernberg). Il semble que le facteur moral discriminant soit la honte. Le premier sous-type est réputé sans vergogne; le second est constamment fragilisé par une quête permanente de l'approbation d'autrui. Enfin, il n'existe aucune thérapie standardisée considérée comme efficace. Tel un serpent de mer, la personnalité narcissique et son trouble ressurgissent chaque fois qu'on met à jour les grandes classifications psychiatriques. Faut-il la conserver? N'est-elle qu'un artefact? Pour le présent propos, il importe surtout de souligner que le narcissisme dont il est question ici n'a plus guère à voir avec le méta-concept obtenu par inférence sur l'analyse des auto-érotismes chez Freud. C'est un ensemble d'attitudes et de traits comportementaux, approché même parfois par la psychologie expérimentale, et qui inspire une confiance mesurée aux théoriciens de la personnalité, tant son objectivation paraît reposer sur des jugements de valeur. En même temps, il y a, dans la littérature professionnelle, un accord plus net sur la spécificité des souffrances des deux types de narcissiques, le grandiose et le vulnérable. Et c'est probablement la raison pour laquelle le trouble de la personnalité narcissique n'a pas été éliminé de la dernière version (le DSM-5) du Manuel statistique et diagnostique de l'Association américaine de psychiatrie, qui sert aujourd'hui de référence internationale. C'est néanmoins la possibilité d'un tel recueil de traits comportementaux, à mi-chemin de la description objectiviste et de l'évaluation moralisante, qui a alimenté et alimente toujours, dans l'essayisme critique de l'individualisme contemporain, un certain nombre de portraits qui revendiquent d'incarner des idéaux-types de la modernité malade. La construction de la personnalité narcissique a probablement été un lieu de formalisation d'une perception particulière du mal-être individualiste. On peut en apercevoir certains aspects avec des instruments épistémologiques et historiques. Mais on ne sait pas grand-chose des institutions qui y ont prêté main forte, des implications médico-légales, des populations spécifiques qui ont été ainsi étiquetées, en bref, de ce qui pourrait servir à une étude de sociologie de la connaissance appliquée à la diffusion de la catégorie. On ne sait pas non plus comment l'approche de la psychologie clinique s'est articulée, ou pas, à la psychanalyse et à la selfpsychology. Quels aspects de la notion freudienne originale ont été jugés féconds, et pourquoi ? On l'ignore. Mais il est difficile de se défendre du sentiment qu'entre le narcissisme de Freud et celui de la critique morale de l'individualisme, il y a autant de rapports, comme dit Spinoza, qu'entre le chien animal aboyant et le Chien constellation.

\section{Une conjecture sur Elias et Cas Wouters}

J'en viens à la partie plus conjecturale de cette étude. Il y a en effet chez Freud une relation étroite entre le narcissisme et le surmoi. Il y a également chez Elias une théorie non psychologique mais socio-historique du surmoi, qui capitalise sur les acquis freudiens pour éclairer le parallélisme entre certaines structures sociales et nos structures psychiques : le surmoi, c'est une des incarnations de l'autocontrainte. Peut-on étendre, dans une veine éliasienne, la même reconstruction sociohistorique en l'appliquant non plus au surmoi mais au narcissisme, en exploitant leur affinité conceptuelle chez Freud ? ${ }^{9}$ L'intérêt de ce rapprochement serait de faire du narcissisme un fait

\footnotetext{
${ }^{8}$ On trouvera un exposé critique lumineux des problèmes sous la plume de Renato Alarcón et Silvana Sarabia, dans «Debates on the Narcissism Conundrum: Trait, Domain, Dimension, Type, or Disorder? », The Journal of Nervous and Mental Disease, vol. 200, $\mathrm{n}^{\circ}$ 1, 2012, p. 625.

${ }^{9}$ Je développe ici une idée formulée initialement par Sabine Delzescaux dans «Autocontrainte et instance surmoïque :
} 
psychologique et moral intrinsèquement lié au processus de civilisation, à l'instar du surmoi au titre de l'autocontrainte. On disposerait alors d'un cadre riche au sein duquel poursuivre l'entreprise de sociologie de la connaissance, de sociologie morale et de sociologie des psychothérapies que j'appelais plus haut de mes vœux. Voici, pas à pas, à quoi ressemblerait l'élaboration d'un tel rapprochement.

Tout d'abord, qu'est-ce qui, chez Freud, articule substantiellement narcissisme et surmoi ? C'est précisément la notion d'idéal du moi. Le surmoi, c'est en fait la face punitive et cruelle de l'idéal du moi quand il se retourne vers le moi et sanctionne, dans sa vie pulsionnelle, toutes les infractions qui font qu'il n'est pas le moi idéal qu'on attend qu'il soit. Dans une névrose "narcissique » comme la mélancolie, par exemple, les auto-accusations peuvent ainsi conduire au suicide : l'idéal terrasse le moi et sa vitalité pulsionnelle en un sentiment d'indignité inexpiable.

Or il n'est pas difficile de remarquer, dans un second temps, que la société de cour, pour prendre le cas-type, articule précisément un très haut degré d'autocontrainte restrictive, interdisant l'expression ouverte de la sexualité et de l'agressivité, avec de puissantes gratifications narcissiques. Car la face positive de cette autocontrainte, c'est l'exaltation d'un moi qui coïncide avec un idéal prestigieux, et qui s'élève vers la grandeur. Dans la société de cour, si j'ose dire, règne le «narcissisme des grandes différences ». Et c'est par là que l'aristocratie en impose. Le sens de l'honneur lié au rang et la honte qui punit quiconque déroge, narcissisent extraordinairement ceux et celles qui en maîtrisent le jeu. Il suffit de penser à La Princesse de Clèves, le premier « roman psychologique » de la littérature française, pour voir les sommets d'amour et de sublimation désexualisante qui s'inventent dans cet univers implacablement hiérarchisé - et le prix payé lors de la chute mélancolique de l'objet. Bien sûr, Elias parle plutôt d'un « idéal du nous » pour exprimer cette pression de classe, la discipline de fer des corps et des âmes qui façonne les courtisans. L'analogie avec l'idéal du moi de Freud n'en est pas moins palpable.

Mais on pourrait peut-être prolonger le parallèle freudien entre surmoi et idéal du moi au-delà de la société de cour. Supposons, pour voir où cela nous mènera, un autre parallèle : entre la diffusion et la généralisation de l'autocontrainte (comme structure psychique surmoïque) et des formes elles aussi plus diffuses et plus démocratiques de l'idéalisation de soi. En un mot, demandons-nous si le « narcissisme des petites différences » du Malaise dans la civilisation n'est pas, en dernière analyse, une extension au grand nombre du «narcissisme des grandes différences ». Freud lui-même fournit des arguments en ce sens, puisqu'à ses yeux le narcissisme des petites différences est l'effet de la trop grande ressemblance des individus entre eux - ce qui se laisse facilement traduire en termes de diminution des inégalités statutaires. Or, ajoute-t-il, les différences peuvent être minuscules, les haines qu'elles causent n'en sont pas moins meurtrières, par un surinvestissement de la différence même au service du narcissisme privé de chacun des membres du groupe. Freud en tire une explication frappante de l'antisémitisme: le narcissisme des petites différences permet l'éconduction collective de l'agressivité au détriment de quelqu'un qui nous ressemble suffisamment, mais cependant pas tout à fait, et dont la petite différence permet au groupe qui se retourne contre lui de trouver un objet pour la haine qui travaille le collectif, alors que l'amour que s'y vouent ses membres plus semblables les uns aux autres tendait plutôt à l'inhiber. En tout état de cause, le narcissisme des petites différences est plutôt une pathologie de l'âge des foules et, à ce titre, une pathologie de la modernité.

L'hypothèse que je soulève est donc celle-ci. Un trait cardinal de l'informalisation des rapports sociaux, selon Wouters, qui prolonge Elias, est l'interdiction d'exprimer ouvertement sa supériorité ou son infériorité, dans un contexte où les chaînes d'interdépendances sociales ne cessent de s'étendre et de proliférer. On comprend alors, dans un tel cadre, que ce qui choque les contempteurs de l'individualisme contemporain, tel Lasch, en particulier le relâchement des codes et certaines formes apparemment complaisantes d'étalement «narcissique » de soi, ne soient en réalité que du

éléments de réflexion sur la référence d'Elias à la psychanalyse freudienne », Nouvelle revue de psychosociologie, vol. 2, n 4, 2007, p. 201-212. 
relâchement hyper-contrôlé. Il est devenu si naturel de s'autocontrôler qu'on n'a plus à se défendre en se «cachant » derrière des conventions formelles. Mais comme Wouters le souligne, le cool a ses règles, et elles ne sont pas moins contraignantes que les règles surmoïques de l'individualisme traditionnel, même si la contrainte consiste désormais à afficher une sorte de non-contrainte. La règle sociale a tout sauf disparu : elle a juste changé de nature.

Or il y a peut-être place ici pour une autre économie du narcissisme congruente avec le narcissisme freudien des petites différences. Car dans cette nouvelle modalité de la contrainte, où la nouvelle «nature » est la contrainte de paraître non-contraint, on ne peut plus se régler sur un idéal du moi transcendant, ni sur des grandeurs ou des supériorités objectives. Si les ajustements mutuels se font entre pairs, cependant, il n'y a pas de raison de croire que les sanctions qu'ils s'infligent quand ils dérogent à la nouvelle règle de non-contrainte soient moins sévères. Chacun, ainsi, devient pour le voisin un idéal du moi et donc aussi, en puissance, un surmoi féroce.

Suivons jusqu'au bout le parallèle que je suggère : l'informalisation se retrouve alors munie d'un complément explicatif des modes d'exclusion, de marginalisation, et peut-être des potentialités ségrégatives qui l'accompagnent implicitement. En somme, l'informalisation serait la face civilisée d'un narcissisme des petites différences qui constituent une "réserve » d'agressivité latente entre quasi-égaux. Et il serait intéressant d'examiner si les pathologies psychologisées sous le chef des troubles de la personnalité narcissique, ou encore borderline, les passages à l'acte explosifs, les attitudes mégalomanes, les effondrements brutaux de l'estime de soi, etc., ne seraient pas plus intelligibles comme des échecs individuels à satisfaire aux normes subtiles et fort coûteuses psychiquement de cette informalisation. Ces patients, vus sous un tel prisme, seraient par exemple les boucs émissaires du groupe de pairs qui les exclut, et leur violence spécifique, morale et parfois physique, doit être déchiffrée comme une exclusion par anticipation, à finalité défensive, de ceux qui les excluent pour leurs infractions fines aux lois de l'autocontrôle contemporain (le relâchement hypercontrôlé).

Comme on le voit, cette approche, tout à fait conjecturale, aurait l'avantage de reconnaître le mérite de la description psychiatrique d'une douleur "narcissique » d'apparition récente, en l'inscrivant donc dans un contexte bien différent de celui de Freud, mais qui, par certains côtés, obéirait quand même à une dynamique socio-historique de l'individualisme susceptible d'attirer l'attention du sociologue éliasien. Il ne suffit pas, en effet, de constater purement et simplement l'hétérogénéité des contenus conceptuels du narcissisme chez Freud, et chez les moralistes critiques de l'individualisme qui s'en approprient des versions très éloignées, sans connaître les controverses sous-jacentes. En réitérant le geste d'Elias, en sociologisant et en historicisant le surmoi freudien, mais avec la notion affine de narcissisme (via l'idéal du moi), on aurait peut-être un moyen d'approcher de façon fructueuse la condition de certains de nos contemporains - sans psychologisme ni moralisme. 